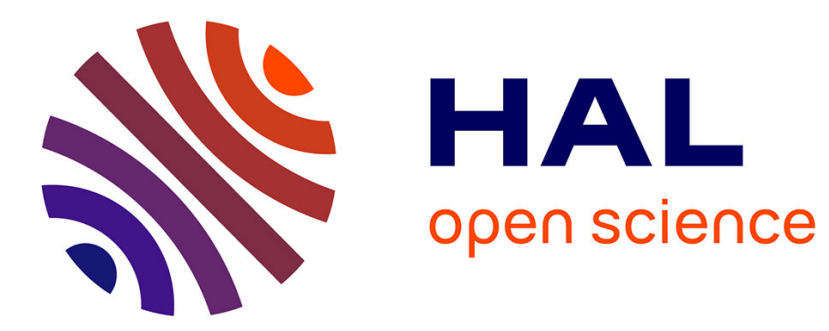

\title{
Output Stabilization of Time-Varying Input Delay Systems Using Interval Observation Technique
}

Andrey Polyakov, Denis Efimov, Wilfrid Perruquetti, Jean-Pierre Richard

\section{To cite this version:}

Andrey Polyakov, Denis Efimov, Wilfrid Perruquetti, Jean-Pierre Richard. Output Stabilization of Time-Varying Input Delay Systems Using Interval Observation Technique. Automatica, 2013, 49 (11), pp.3402-3410. 10.1016/j.automatica.2013.08.012 . hal-00847565

\section{HAL Id: hal-00847565 \\ https://inria.hal.science/hal-00847565}

Submitted on 23 Jul 2013

HAL is a multi-disciplinary open access archive for the deposit and dissemination of scientific research documents, whether they are published or not. The documents may come from teaching and research institutions in France or abroad, or from public or private research centers.
L'archive ouverte pluridisciplinaire HAL, est destinée au dépôt et à la diffusion de documents scientifiques de niveau recherche, publiés ou non, émanant des établissements d'enseignement et de recherche français ou étrangers, des laboratoires publics ou privés. 


\title{
Output Stabilization of Time-Varying Input Delay Systems Using Interval Observation Technique
}

\author{
Andrey Polyakov ${ }^{\mathrm{a}, \mathrm{c}}$, Denis Efimov ${ }^{\mathrm{a}, \mathrm{b}, \mathrm{d}}$,Wilfrid Perruquetti ${ }^{\mathrm{b}, \mathrm{a}}$, \\ Jean-Pierre Richard ${ }^{\mathrm{b}, \mathrm{a}}$ \\ ${ }^{a}$ Non-A INRIA - LNE, Parc Scientifique de la Haute Borne 40, av. Halley Bat.A, Park Plaza 59650 Villeneuve d'Ascq, France \\ ${ }^{\mathrm{b}}$ Ecole Centrale de Lille and LAGIS UMR CNRS 8219, BP 48, Cité Scientifique, 59651 Villeneuve-d'Ascq, France \\ ${ }^{\mathrm{c}}$ Institute of Control Sciences of Russian Academy of Sciences, 117997, Profsouyznaya 65, Moscow, Russia \\ ${ }^{\mathrm{d}}$ Department Control Systems and Informatics, Saint-Petersburg National Research University of Information Technologies, \\ Mechanics and Optics, 197101, 49 Kronverkskiy pr., 49, St. Petersburg, Russia.
}

\begin{abstract}
The output stabilization problem for a linear system with an unknown bounded time-varying input delay is considered. The interval observation technique is applied in order to obtain guaranteed interval estimate of the system state. The procedure of the interval observer synthesis uses lower and upper estimates of the unknown delay and requires to solve a special Silvester's equation. The interval predictor is introduced in order to design a linear stabilizing feedback. The control design procedure is based on Linear Matrix Inequalities (LMI). The theoretical results are supported by numerical simulations and compared with a control design scheme based on a Luenberger-like observer.
\end{abstract}

\section{Introduction and related works}

A time-varying input delay arises in models of control systems due to many reasons. Usually its presence is motivated by a physical nature of a plant. It may be related to transport delays (like in chemical, hydraulic or pneumatic systems) or computational delays (e.g. in digital controllers or communication networks [17]). On the other hand, time-varying input delay can be introduced "artificially" in order to model a sampling effect (see, for example, [26], [12], [11]).

Control of a system with input delay is an important problem treated in literature (see, for example, [23], [16],

\footnotetext{
^ This work was supported by the European INTERREG IV A 2 Mers Seas Zeen Cross-border Cooperation Program 2007-2013 under SYSIASS 6-20 project. It was also supported by Ministry of Higher Education and Research, NordPas de Calais Regional Council and FEDER through the Contrat de Projets Etat Region (CPER) CIA 2007-2013. Corresponding author A. Polyakov.

Email addresses: andrey.polyakov@inria.fr (Andrey Polyakov), denis.efimov@inria.fr (Denis Efimov), wilfrid.perruquetti@inria.fr (Wilfrid Perruquetti), jean-pierre.richard@ec-lille.fr (Jean-Pierre Richard).
}

[19] and references within). The predictor-based feedback [25] is a usual tool for control design if the input delay is known. This method is well-developed for both constant and time-varying delays [1], [28], [16]. It has been effectively used even for nonlinear [3] and sliding mode control systems [21]. If delay is constant, but unknown, then delay estimation technique [4] and/or the delay-adaptive control approach [5] can be applied. For unknown delay the predictor-based feedback design has to be accompanied with robustness analysis [16], [29].

Typically, the predictor feedback is effectively applicable if the whole state-vector of a system is measured [25], [23], [29], [16], [21]. The observer design for systems with time-varying input and state delay is presented in [24]. The results related to designing of an output predictor feedback for systems with input delays, which are known and constant, can be found, for example, in [27] and [15]. The adaptive output feedback regulator for a chain of integrators with an unknown time-varying delay in the input is presented in [6].

In this paper a recently developed technique of interval observers [13], [18], [22] is applied in order to tackle the problem of the output-based control design for linear MIMO systems with unknown time-varying input delay. 
The interval observer provides the guaranteed interval estimates of the system state in a real-time. This property simplifies the control of transition processes with respect to system state [8]. The problem of an interval observer design was studied in [20] for systems with known delays and in [7] for systems with unknown state delays. The present paper extends the interval observer technique to systems with unknown time-varying input delay under assumption that lower and upper estimates of the delay are given. It also presents the interval prediction scheme (interval predictor), that allows us to realize a feedback design based on LMI.

\section{Notations}

- The set of real numbers is denoted by $\mathbb{R}$ and $\mathbb{R}_{+}:=$ $\{x \in \mathbb{R}: x>0\}$.

- $\operatorname{spec}(A)$ is the spectrum of the matrix $A \in \mathbb{R}^{n \times n}$.

- $\Re(\lambda)$ is the real part of the complex number $\lambda$.

- $\mathbb{H}$ is the set of Hurwitz matrices from the set $\mathbb{R}^{n \times n}$, i.e. $A \in \mathbb{H} \quad \Leftrightarrow \quad \Re(\lambda)<0$ for all $\lambda \in \operatorname{spec}(A)$.

- $\mathbb{M}$ is the set of Metzler matrices from the set $\mathbb{R}^{n \times n}$, i.e. $R=\left\{r_{i j}\right\}_{i, j=1}^{n} \in \mathbb{M} \quad \Leftrightarrow \quad r_{i j} \geq 0$ for $i \neq j$.

- The inequality $F \succ 0(F \prec 0)$ for a symmetric matrix $F \in \mathbb{R}^{n \times n}$ denotes its positive (negative) definiteness. The order relations $F \succeq 0$ and $F \preceq 0$ are used in order to assign the positive and negative semidefiniteness of the matrix $F$, respectively.

- The inequalities $x>0, x<0, x \geq 0$ and $x \leq 0$ written for the vector $x \in \mathbb{R}^{n}$ are understood in a componentwise sense. The inequalities $A>0, A<0$, $A \geq 0$ and $A \leq 0$ written for the matrix $A \in \mathbb{R}^{n \times m}$ are understood analogously.

- The identity matrix of the size $n \times n$ is denoted by $I_{n}$; the square zero matrix of the size $n \times n$ is denoted by $0_{n}$; the rectangular zero matrix of the size $n \times m$ is denoted by $0_{n \times m}$.

\section{Problem statement}

Consider the input delay control system of the form

$$
\left\{\begin{array}{l}
\dot{x}(t)=A x(t)+B u(t-h(t)), \quad t>0, \\
y(t)=C x(t),
\end{array}\right.
$$

where $x \in \mathbb{R}^{n}$ is the system state, $u \in \mathbb{R}^{m}$ is the vector of control inputs, $y \in \mathbb{R}^{k}$ is the measured output, $A \in$ $\mathbb{R}^{n \times n}, B \in \mathbb{R}^{n \times m}$ and $C \in \mathbb{R}^{k \times n}$ are known matrices and the input delay $h(t)$ is assumed to be an unknown locally integrable function within the bounded interval:

$$
0 \leq \underline{h} \leq h(t) \leq \bar{h}
$$

where the numbers $\underline{h}$ and $\bar{h}$ are given. The system (1) is studied with the initial conditions:

$$
x(0)=x_{0}, \quad u(t)=v(t) \text { for } t \in[-\bar{h}, 0),
$$

where $v(t)$ is some continuous function.

Assumption 1 The pair $(A, B)$ is controllable and the $\operatorname{pair}(A, C)$ is observable.

Assumption 2 For any $t \geq 0$ the information on the control signal $u(t)$ on the time interval $[t-\bar{h}, t)$ can be stored and used for control design purposes.

Assumption 3 The set $\Omega \subset \mathbb{R}^{n}$ of admissible initial conditions $x_{0} \in \Omega$ of the system (1) is assumed to be bounded and known.

Remark that the second assumption is usual for a predictor-based approach to control design.

The main objective of this paper is to design a control algorithm for exponential stabilization of the system (1), i.e. for some numbers $c, r>0$ any solution of the closedloop system (1) has to satisfy the inequality $\|x(t)\| \leq$ $c e^{-r t}, \forall t>0$, where $x(0) \in \Omega$.

\section{Interval observer and interval predictor de- sign}

\subsection{Interval observer}

Let us introduce the following notations

$$
\begin{aligned}
& \underline{B^{\prime} u}(\tau):=\min _{\theta \in[0, \bar{h}-\underline{h}]} B^{\prime} u(\tau-\theta), \\
& \overline{B^{\prime} u}(\tau):=\max _{\theta \in[0, \bar{h}-\underline{h}]} B^{\prime} u(\tau-\theta),
\end{aligned}
$$

where $\min (\max )$ is considered componentwise and $B^{\prime}$ is some matrix of an appropriate dimension.

Since the input delay of the system (1) is unknown then formally we need to estimate the state of the system with unknown input. However, for the case of bounded delay under assumption 2 we may design the so-called interval observer using lower and upper estimates of the input signals over the delay interval $[t-\bar{h}, t-\underline{h}]$.

Lemma 4 Under Assumptions 1-3 there always exist matrices $L \in \mathbb{R}^{n \times k}$ and $S \in \mathbb{R}^{n \times n}$, $\operatorname{det}(S) \neq 0$ such that

$$
A+L C \in \mathbb{H}, \quad S^{-1}(A+L C) S \in \mathbb{M},
$$

such that for any continuous control signal $u: \mathbb{R}_{+} \rightarrow \mathbb{R}^{m}$ the interval observer of the form

$$
\begin{aligned}
& \dot{\dot{x}}(t)=\tilde{A} \underline{x}(t)+\underline{\tilde{B} u}(t-\underline{h})+\tilde{L}(\tilde{C} \underline{x}(t)-y(t)), \\
& \dot{\bar{x}}(t)=\tilde{A} \bar{x}(t)+\tilde{\tilde{B}} u(t-\underline{h})+\tilde{L}(\tilde{C} \bar{x}(t)-y(t)), \\
& \tilde{A}=S^{-1} A S, \tilde{B}=S^{-1} B, \tilde{L}=S^{-1} L, \tilde{C}=C S
\end{aligned}
$$


has the following properties:

1) if $\underline{x}(0) \leq \tilde{x}(0) \leq \bar{x}(0)$ then $\underline{x}(t) \leq \tilde{x}(t) \leq \bar{x}(t)$ for $t>0$, where

$$
\tilde{x}(t)=S^{-1} x(t)
$$

and $x(t)$ is the solution of the system (1), (3) with $x_{0} \in \Omega$;

2) if $\exists \Delta^{*} \in \mathbb{R}_{+}^{n}$ and $\exists q \in \mathbb{R}_{+} \cup\{0\}$ such that

$$
\overline{\tilde{B} u}(t-\underline{h})-\underline{\tilde{B} u}(t-\underline{h}) \leq \Delta^{*} e^{-q t} \text { for } t>0 \in \mathbb{R}_{+},
$$

then for all $p \in\left(0, \min _{\lambda \in \operatorname{spec}(\tilde{A}+\tilde{L} \tilde{C})}-\Re(\lambda)\right)$ there exists a matrix $M \in \mathbb{R}_{+}^{n \times n}$ such that

$$
\begin{gathered}
\tilde{x}(t)-\underline{x}(t) \leq M e^{-p t}\left(\tilde{x}(0)-\underline{x}(0)+\nu(p-q, t) \Delta^{*}\right), \\
\bar{x}(t)-\tilde{x}(t) \leq M e^{-p t}\left(\bar{x}(0)-\tilde{x}(0)+\nu(p-q, t) \Delta^{*}\right), \\
\text { where } \nu(p-q, t)=\left\{\begin{array}{cl}
\frac{e^{(p-q) t}-1}{p-q} & \text { for } p \neq q, \\
t & \text { for } p=q .
\end{array}\right.
\end{gathered}
$$

Proof. 1) Since the pair $(A, C)$ is observable then an appropriate selection of the matrix $L$ can assign any real simple negative spectrum to the matrix $A+L C$, i.e. $\sigma(A+L C)=\left\{\lambda_{i}\right\}_{i=1}^{n}, \lambda_{i}<0$ and $\lambda_{i} \neq \lambda_{j}$ for $i \neq j$. Then the matrix $S$ can be defined as Jordan transformation (see, for example, [18]) for $A+L C$, which is real in this case. Indeed, $S^{-1}(A+L C) S=\operatorname{diag}\left(\lambda_{i}\right) \in \mathbb{M} \cap \mathbb{H}$. Other variants of computation of $S$ and $L$ are also possible [22].

Since the control signal $u$ is continuous and the delay function $h$ is locally Lebesgue integrable then the function $u(t-h(t))$ is locally Lebesgue integrable and all solutions of the system (1), (3) are defined for all $t>0$. The assumption 2 implies that the functions $u(t-\underline{h})$, $\underline{\tilde{B} u}(t-\underline{h})$ and $\overline{\tilde{B} u}(t-\underline{h})$ can be calculated for any $t \geq 0$. So, the interval observer (7) is correctly defined. Denote $\underline{e}=\tilde{x}-\underline{x}$ and $\bar{e}=\bar{x}-\tilde{x}$. In this case we have

$$
\begin{aligned}
& \underline{\dot{e}}=(\tilde{A}+\tilde{L} \tilde{C}) \underline{e}+\tilde{B} u(t-h(t))-\underline{\tilde{B} u}(t-\underline{h}), \\
& \dot{\bar{e}}=(\tilde{A}+\tilde{L} \tilde{C}) \bar{e}+\overline{\tilde{B}} u(t-\underline{h})-\tilde{B} u(t-h(t)),
\end{aligned}
$$

where $\tilde{A}+\tilde{L} \tilde{C} \in \mathbb{M} \cap \mathbb{H}$. Since $\tilde{B} u(t-h(t))-\underline{\tilde{B} u}(t-\underline{h}) \geq 0$ and $\bar{B} u(t-\underline{h})-\tilde{B} u(t-h(t)) \geq 0$, then the system (8) is positive [9]. Therefore, the inequalities $\underline{e}(0) \geq 0$, $\bar{e}(0) \geq 0$ imply $\underline{e}(t) \geq 0, \bar{e}(t) \geq 0$ for all $t>0$, i.e. the first statement of the lemma is proven.

2) The matrix $\tilde{A}+\tilde{L} \tilde{C}$ is Metzler and Hurwitz. So, $e^{t(\tilde{A}+\tilde{L} \tilde{C})} \geq 0$ and for any $p \in\left(0, \min _{\lambda \in \operatorname{spec}(\tilde{A}+\tilde{L} \tilde{C})}-\Re(\lambda)\right)$ we have $\tilde{A}+\tilde{L} \tilde{C}+p I_{n} \in \mathbb{H}$. Hence, there exists $M \in \mathbb{R}_{+}^{n \times n}$ such that the inequality $e^{t(\tilde{A}+\tilde{L} \tilde{C})} \leq M e^{-p t}$ holds and results the following estimates

$$
\begin{gathered}
\underline{e}(t) \leq M e^{-p t} \underline{e}(0)+ \\
M \int_{0}^{t} e^{p(s-t)}(\tilde{B} u(s-h(s))-\underline{\tilde{B} u}(s-\underline{h})) d s, \\
\bar{e}(t) \leq M e^{-p t} \bar{e}(0)+ \\
M \int_{0}^{t} e^{p(s-t)}(\overline{\tilde{B} u}(s-\underline{h})-\tilde{B} u(s-h(s))) d s .
\end{gathered}
$$

Finally, the inequalities

$$
\begin{aligned}
& \tilde{B} u(t-h(t))-\underline{\tilde{B} u}(t-\underline{h}) \leq \overline{\tilde{B} u}(t-\underline{h})-\underline{\tilde{B} u}(t-\underline{h}), \\
& \overline{\tilde{B} u}(t-\underline{h})-\tilde{B} u(t-h(t)) \leq \overline{\tilde{B}} u(t-\underline{h})-\underline{\tilde{B}} u(t-\underline{h})
\end{aligned}
$$

imply the second statement of the lemma.

Lemma 4 guarantees that a bounded control input always implies a bounded observation error of the observer (7). Moreover, if $u(t)$ converges to some constant value exponentially with the rate $q \in \mathbb{R}_{+}$then the observation errors of the interval observer (7) converge to zero exponentially with the rate $\min \{p, q\}$, where $p \in\left(0, \min _{\lambda \in \operatorname{spec}(\tilde{A}+\tilde{L} \tilde{C})}-\Re(\lambda)\right)$. Theorem 8 below uses this property in order to prove the exponential convergence of the observed states to the real ones.

Remark 5 To realize in practice the interval observer (7) the condition $x(0) \leq \tilde{x}(0) \leq \bar{x}(0)$ must be guaranteed. Since the set of admissible initial conditions $\Omega$ is assumed to be known (see, Assumption 3), the required inequality can be ensured. For example, if $\Omega=\left\{x \in \mathbb{R}^{n}: x^{T} P x<\right.$ $1\}, P \succ 0$, then $\tilde{x}^{T} S^{T} P S \tilde{x}<1$ and $\underline{x}_{i}(0)=-\bar{x}_{i}(0)=$ $-1 / \lambda_{\min }\left(S^{T} P S\right), i=1,2, \ldots, n$. Similar estimates can be also presented if $\Omega$ is a polyhedron.

Let some Hurwitz and Metzler matrix $R$ be given and suppose we need to find $S$ and $L$ such that

$$
S^{-1}(A+L C) S=R
$$

Denote $X=S^{-1}$ and $Y=S^{-1} L$. In this case the required equality can be rewritten in the form of Silvester's equation [2]

$$
X A+Y C=R X,
$$

where $X \in \mathbb{R}^{n \times n}$ and $Y \in \mathbb{R}^{n \times k}$.

Proposition 6 [2], [22] If the matrix $R$ has disjoint spectrum and the pair $(A, C)$ is observable then the equation (9) has a solution. 
Equation (9) can be rewritten in the form of the system of linear algebraic equations

$$
W z=0,
$$

where $W=\left(I_{n} \otimes A^{T}-R \otimes I_{n} I \otimes C^{\prime}\right)$, $z=\left(x_{11}, \ldots, x_{n 1}, x_{21}, \ldots, x_{n n}, y_{11}, \ldots, y_{n 1}, y_{21}, \ldots, y_{n k}\right)^{T}$, and $\otimes$ is the Kroneker product. So, numerically the required solution of the equation (9) can be found as an element of the null space of the matrix $W$.

\subsection{Interval Predictor}

Consider the system (7). By analogy with Artstein transformation [1] let us introduce the following predictor variables:

$$
\begin{aligned}
& \underline{z}(t)=e^{\tilde{A} \underline{h}} \underline{x}(t)+\int_{-\underline{h}}^{0} e^{-A \theta} \underline{\tilde{B} u}(t+\theta) d \theta, \\
& \bar{z}(t)=e^{\tilde{A}} \underline{h} \bar{x}(t)+\int_{-\underline{h}}^{0} e^{-A \theta} \overline{\tilde{B} u}(t+\theta) d \theta
\end{aligned}
$$

which are correctly defined due to Assumption 2. The introduced variables estimate the interval observer states at the future time moment $t+\underline{h}$, i.e. $\underline{z}(t) \approx \underline{x}(t+\underline{h})$ and $\bar{z}(t) \approx \bar{x}(t+\underline{h})$. These approximate equalities become exact if the observation error tends to zero (see, $(7)$ ).

Below it is shown that a stabilizing control for the original system can be designed as a linear feedback with respect to the predictor variables.

Remark 7 In order to compare the interval observation approach with a scheme based on the usual Luenbergerlike observation technique we also consider the following observer

$$
\dot{\hat{x}}(t)=A \hat{x}(t)+B u(t-\underline{h})+\mathcal{L} C(\hat{x}(t)-y(t)),
$$

where $\hat{x} \in \mathbb{R}^{n}$ is the observer state and the matrix $\mathcal{L} \in \mathbb{R}^{n \times k}$ is such that $A+\mathcal{L} C \in \mathbb{H}$. Analogously to Lemma 4 it can be easily proven the similar Input-to-State stability property for the presented observer, namely, if $\exists \Delta^{*} \in \mathbb{R}_{+}$and $\exists q \in \mathbb{R}_{+}$:

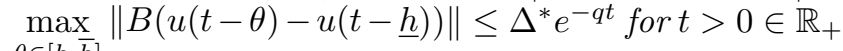
$\theta \in[\underline{h}, \bar{h}]$

then for any $p \in\left(0, \min _{\lambda \in \operatorname{spec}(A+\mathcal{L} C)}-\Re(\lambda)\right)$ there exists $M \in \mathbb{R}_{+}$such that

$$
\|\hat{x}(t)-x(t)\| \leq M e^{-p t}\left(\|\hat{x}(0)-x(0)\|+\nu(p-q, t) \Delta^{*}\right),
$$

where $\nu(p-q, t)$ is defined in the statement of Lemma 4. The corresponding predictor variable $\hat{z}(t)$ is

$$
\hat{z}(t)=e^{A \underline{h}} \hat{x}(t)+\int_{-h}^{0} e^{-A \theta} B u(t+\theta) d \theta .
$$

\section{$5 \quad$ Stabilizing control design}

Assume that the interval observer (7) for the system (1) is designed and the matrices $S, \tilde{A}, \tilde{L}, \tilde{C}, \tilde{B}$ are obtained.

Let us define the control in the form

$$
u(t)=K z(t), \quad z(t)=\frac{1}{2}(\bar{z}(t)+\underline{z}(t)),
$$

where the matrix $K \in \mathbb{R}^{m \times n}$ is to be designed.

Let $\tilde{B}_{i} \in \mathbb{R}^{n \times m}, i=1,2, \ldots, n$ be the matrix such that $i$-th row of $\tilde{B}_{i}$ coincides with $i$-th row of the matrix $\tilde{B}$ but all other rows of $\tilde{B}_{i}$ are zero. Denote also $\tilde{B}_{n+i}=$ $\tilde{B}_{i}, i=1,2 \ldots, n$.

Theorem 8 If for some given $\alpha \in \mathbb{R}_{+}$the matrices $X, Z, R_{i}, S_{i} \in \mathbb{R}^{n \times n}, i=1,2, \ldots, 2 n$ and the matrix $Y \in$ $\mathbb{R}^{m \times n}$ satisfy the following LMI system

$$
\left(\begin{array}{cc}
W_{e} & W_{e z} \\
W_{e z}^{T} & W_{z}
\end{array}\right) \prec 0, X \succ 0, Z \succ 0, R_{i} \succ 0, S_{i} \succ 0,
$$

where

$$
\begin{aligned}
& W_{e}=\left(\begin{array}{cccc}
\Pi_{1} & \tilde{B}_{1} Y & \ldots & \tilde{B}_{2 n} Y \\
Y^{T} \tilde{B}_{1}^{T} & -e^{-\alpha \Delta h} S_{1} & \ldots & 0_{n} \\
\ldots & \ldots & \ldots & \ldots \\
Y^{T} \tilde{B}_{2 n}^{T} & \ldots & \ldots & -e^{-\alpha \Delta h} S_{2 n}
\end{array}\right), \\
& W_{z}=\left(\begin{array}{ccccc}
\Pi_{2} & \Pi_{23} & \tilde{B}_{1} Y & \ldots & \tilde{B}_{2 n} Y \\
\Pi_{23}^{T} & \Pi_{3} & \tilde{B}_{1} Y & \ldots & \tilde{B}_{2 n} Y \\
Y^{T} \tilde{B}_{1}^{T} & Y^{T} \tilde{B}_{1}^{T} & -e^{-\alpha \Delta h} R_{1} & \ldots & 0_{n} \\
\ldots & \ldots & \ldots & \ldots & \ldots \\
Y^{T} \tilde{B}_{2 n}^{T} & Y^{T} \tilde{B}_{2 n}^{T} & 0_{n} & \ldots & -e^{-\alpha \Delta h} R_{2 n}
\end{array}\right), \\
& W_{e z}=\left(\begin{array}{cc}
Z \tilde{C}^{T} \tilde{L}^{T} e^{\tilde{A}^{T} \underline{h}}\left[\begin{array}{cc}
I_{n} & I_{n}
\end{array}\right] & 0_{n \times 2 n^{2}} \\
0_{2 n^{2} \times 2 n} & 0_{2 n^{2}}
\end{array}\right), \\
& \Pi_{1}=(\tilde{A}+\tilde{L} \tilde{C}) Z+Z(\tilde{A}+\tilde{L} \tilde{C})^{T}+\alpha Z, \\
& \Pi_{2}=\tilde{A} X+\tilde{B} Y+X \tilde{A}^{T}+Y^{T} \tilde{B}^{T}+\alpha X, \\
& \Pi_{3}=\frac{1}{4} \sum_{i=1}^{2 n}\left(R_{i}+e^{\alpha \underline{h}} S_{i}\right)-\frac{2}{\Delta h} X, \Delta h:=\bar{h}-\underline{h}, \\
& \Pi_{23}=X \tilde{A}^{T}+Y^{T} \tilde{B}^{T},
\end{aligned}
$$

then

1) the system (1) together with the control (15) for $K=$ $Y X^{-1}$ is exponentially stable with the convergence rate greater than or equals to $\alpha / 2$;

2) the states $x(t)$ and $\bar{x}(t)$ of the observer (7) tends to $\tilde{x}(t)=S^{-1} x(t)$ exponentially with the convergence rate greater than or equals to $\alpha / 2$. 
Proofs of all theorems are given in the Appendix.

In fact, the parameter $\alpha \in \mathbb{R}_{+}$has to be selected as follows

$$
0<\alpha<-2 \max _{\lambda \in \operatorname{spec}(\tilde{A}+\tilde{L} \tilde{C})} \Re(\lambda) .
$$

Indeed, the necessary condition for feasibility of the LMI system (16) is $\Pi_{1} \prec 0$, which is equivalent to $\tilde{A}+\tilde{L} \tilde{C}+$ $0.5 \alpha I_{n} \in \mathbb{H}$. Since $\tilde{A}+\tilde{L} \tilde{C} \in \mathbb{H}$ then the selection of the positive parameter $\alpha$ only from the mentioned interval guarantees $\Pi_{1} \prec 0$.

Under conditions of controllability of the pair $\{A, B\}$ the LMI system (16) is feasible at least for sufficiently small $\Delta h$. Indeed, if we select $R_{i}=S_{i}=\frac{1}{\sqrt{\Delta h}} X, i=1,2, \ldots, 2 n$ then for $\Delta h \rightarrow 0$ we easily derive that feasibility of (16) follows from feasibility of

$$
\left(\begin{array}{cc}
\Pi_{1} & Z \tilde{C}^{T} \tilde{L}^{T} e^{\tilde{A}^{T} \underline{h}} \\
e^{\tilde{A}} \underline{h} \tilde{L} \tilde{C} Z & \Pi_{2}
\end{array}\right) \prec 0, X \succ 0, Z \succ 0 .
$$

The last LMI system is always feasible if Assumption 1 holds.

Remark 9 Let us mention that the control function can be selected in a more general form $u(t)=K z(t)+\bar{K} \bar{z}(t)$, where $\underline{K}, \bar{K} \in \mathbb{R}^{m \times n}$. In particular, the gain matrices can be defined as $\underline{K}=\mu K$ and $\bar{K}=(1-\mu) K$, where $\mu \in$ $[0,1]$. This form of control implies some small changes in formulation and proof of Theorem 8. We select $\mu=0.5$ for simplicity and shortness. Moreover, such selection had allowed us to attain the best convergence rate during numerical simulations.

Similarly to Theorem 8 the following result can be provided for output stabilization of time-varying input delay system (1) using Luenberger-like observer (13).

Theorem 10 If the following system of bilinear matrix inequalities

$$
\begin{gathered}
\mathcal{W}:=\left(\begin{array}{cccc}
\mathcal{W}_{11} & \mathcal{W}_{21}^{T} & \mathcal{W}_{21}^{T} & B \mathcal{Y} \\
\mathcal{W}_{21} & \mathcal{W}_{22} & \mathcal{W}_{23} & 0 \\
\mathcal{W}_{21} & \mathcal{W}_{23}^{T} & \mathcal{W}_{33} & 0 \\
\mathcal{Y}^{T} B^{T} & 0 & 0 & -e^{-\alpha \bar{h}} \mathcal{R}
\end{array}\right) \prec 0, \quad(17) \\
\mathcal{W}_{11}=A \mathcal{Z}+\mathcal{Z} A^{T}+\mathcal{L} C \mathcal{Z}+\mathcal{Z} C^{T} \mathcal{L}^{T}+\alpha \mathcal{Z}, \quad \mathcal{Z} \succ 0, \\
\mathcal{W}_{22}=A \mathcal{X}+\mathcal{X} A^{T}+B \mathcal{Y}+\mathcal{Y}^{T} B^{T}+\alpha \mathcal{X}, \quad \mathcal{X} \succ 0, \\
\mathcal{W}_{21}=e^{A} \underline{L} \mathcal{L}, \mathcal{W}_{23}=A \mathcal{X}+B \mathcal{Y}, \mathcal{W}_{33}=\mathcal{R}-\frac{1}{\Delta h} \mathcal{X}, \\
\alpha \in \mathbb{R}_{+}, \mathcal{Z}, \mathcal{X}, \mathcal{R} \in \mathbb{R}^{n \times n}, \mathcal{Y} \in \mathbb{R}^{m \times n}, \mathcal{L} \in \mathbb{R}{ }^{n \times k}, \mathcal{R} \succ 0, \\
\text { is feasible then the system }(1) \text { with the control } \\
u(t)=\mathcal{K} \hat{z}(t),
\end{gathered}
$$

where $z_{l}(t)$ is defined by (14), (13) and $\mathcal{K}=\mathcal{Y} \mathcal{X}^{-1}$, is exponentially stable with the convergence rate greater than or equals to $\alpha / 2$.

If the observer gain $\mathcal{L} \in \mathbb{R}^{n \times k}$ is selected such that $A+$ $\mathcal{L} C \in \mathbb{H}$ then the matrix inequality (17) becomes LMI with respect to the rest of variables. The obtained LMI is feasible at least for sufficiently small $\Delta h$. The proof of this fact repeats the considerations presented after the statement of Theorem 8 .

The control design schemes introduced in Theorems 8 and 10 are based on prediction technique (see, [1], [16]), which allows us to "compensate" the input delay up to the lower known boundary $\underline{h}$. So, feasibility of the corresponding matrix inequalities is mainly depended on $\Delta h$.

\section{Example}

\subsection{Linear oscillator}

Consider the system (1) with $n=2, k=m=1$ and parameters

$$
A=\left(\begin{array}{cc}
0 & 1 \\
-1 & 0
\end{array}\right), B=\left(\begin{array}{l}
0 \\
1
\end{array}\right), C=\left(\begin{array}{ll}
1 & 0
\end{array}\right) .
$$

In order to compare the control schemes based on the interval observation method and the Luenberger-like observer design we fix the lower boundary of the input delay $\underline{h}=1$ and try to find maximum possible upper boundary delay $\bar{h}$ for each scheme. In both cases we try to design the control, which guarantees the exponential convergence rate $5 \cdot 10^{-4}$, i.e. $\alpha=0.001$.

For the control design using interval observer technique the maximum upper bound of the delay is found $\bar{h}=$ 2.037. The observer parameters are selected by means of solving the Silvester's equation (9) for

It leads to

$$
R=\left(\begin{array}{cc}
-63.95825 & 8.60475 \\
0.7077 & -1.49000
\end{array}\right)
$$

$$
\begin{gathered}
S=\left(\begin{array}{cc}
-65.43359 & 13.91293 \\
-87.64917 & 326.80682
\end{array}\right), \tilde{L}=10^{-2}\left(\begin{array}{c}
99.98522 \\
-0.17489
\end{array}\right), \\
\tilde{A}=\left(\begin{array}{cc}
1.46567 & -5.30612 \\
0.59331 & -1.46566
\end{array}\right), \tilde{B}=10^{-3}\left(\begin{array}{l}
0.68996 \\
3.24496
\end{array}\right), \\
\tilde{C}=\left(\begin{array}{ll}
-65.43359 & 13.91293
\end{array}\right) .
\end{gathered}
$$

Finally, using Sedumi-1.3 for MATLAB we solve LMI system (16) and obtain

$$
K=(60.34248-299.16907) \text {. }
$$

For an interval observer design it is assumed that $x(0) \in$ $\left\{x \in \mathbb{R}^{2}:\left|x_{i}\right| \leq 1, i=1,2\right\}$. Then due to relation $\tilde{x}(0)=$ $S^{-1} x(0)$ the initial conditions for the interval observer 


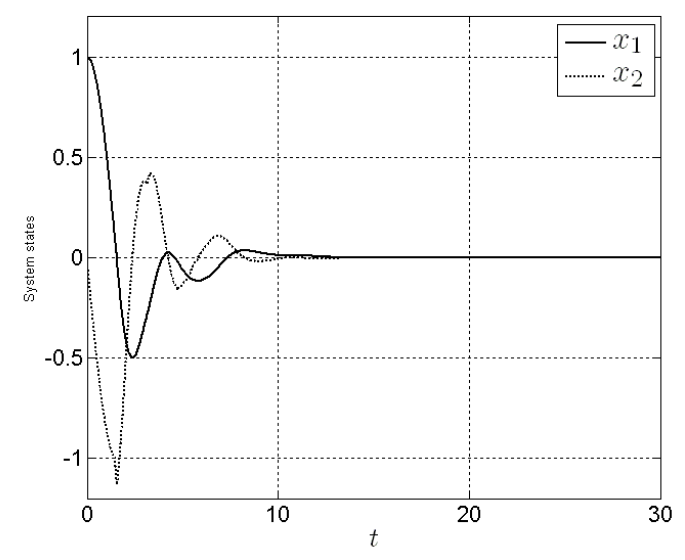

Fig. 1. Evolution of the system states for the interval observer-based control scheme

(7) can be selected as $\bar{x}(0)=\left|S^{-1}\right|\left(\begin{array}{l}1 \\ 1\end{array}\right), \underline{x}(0)=-\bar{x}(0)$, where the modulus of the matrix is understood componentwise.

For the Luenberger-like observer-based control scheme the maximum upper bound of the input delay is found $\bar{h}=1.464$. The corresponding observer gain is

$$
\mathcal{L}=\left(\begin{array}{l}
-1.07670 \\
-0.31524
\end{array}\right) \text {. }
$$

Using Sedumi-1.3 for MATLAB we solve the LMI system (17) with selected $\mathcal{L}$ and obtain

$$
\mathcal{K}=(0.138642-1.077025)
$$

Remark, if for Luenberger-like observer we use the same gain $\mathcal{L}=S \tilde{L}$ as for the interval one then the maximum upper boundary of the input delay, for which LMI (17) is feasible, is tangibly less than 1.464 .

The figures 1 and 2 depict the results of numerical simulations for the system (1) with the control (15) and the control (18), respectively, with the following initial conditions: $x(0)=(0,1)^{T}$ and $v(t)=0$. In both cases the delay function is defined by the following formula $h(t)=0.5(\bar{h}+\underline{h})+0.5(\bar{h}-\underline{h}) \operatorname{sign}(\sin (2 t)))$. The simulations have done using the explicit Euler method with a constant step size $\tau=0.01$. They show the better control performance for the interval observer-based control scheme.

\subsection{Double integrator}

The adaptive control scheme presented in [6] also deals with an unknown time varying input delay, but it is applicable only for a chain of integrators. In order to compare our control algorithms with the one presented in [6] we consider the output control problem for double integrator, i.e. $n=2, k=m=1$ and

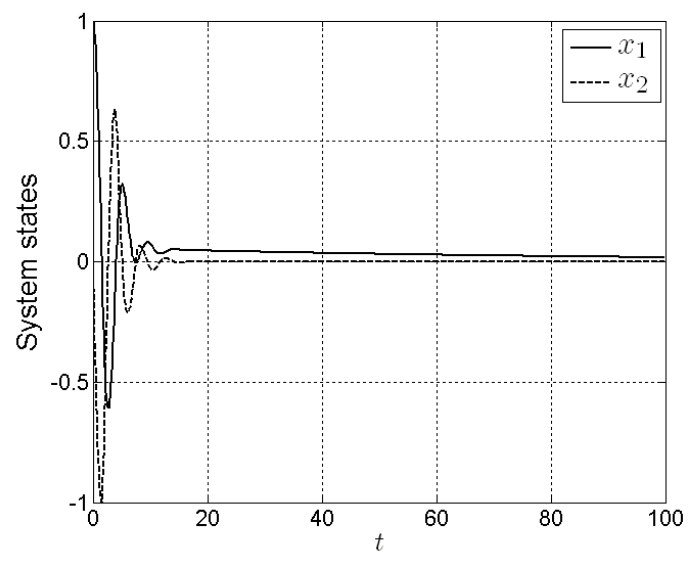

Fig. 2. Evolution of the system states for the Luenberger-like observer-based control scheme

$$
A=\left(\begin{array}{ll}
0 & 1 \\
0 & 0
\end{array}\right), B=\left(\begin{array}{l}
0 \\
1
\end{array}\right), C=\left(\begin{array}{ll}
1 & 0
\end{array}\right) .
$$

For the control scheme based on interval observation we solve the Silvester's equation (9) with

and derive

$$
R=\left(\begin{array}{cc}
-3.0000 & 2.3200 \\
0.2700 & -0.4100
\end{array}\right)
$$

$$
\begin{gathered}
S=\left(\begin{array}{cc}
-3.6234 & -0.2599 \\
-1.5558 & -9.1860
\end{array}\right), \tilde{L}=\left(\begin{array}{c}
0.9479 \\
-0.0948
\end{array}\right) \\
\tilde{A}=\left(\begin{array}{cc}
0.4346 & 2.5663 \\
-0.0736 & -0.4346
\end{array}\right), \tilde{B}=\left(\begin{array}{c}
0.0079 \\
-0.1102
\end{array}\right), \\
\tilde{C}=(-3.6234-0.2599) .
\end{gathered}
$$

The numerical simulations have been done for the same time delay as in [6] : i) $h(t)=2 \sin (t)+2$; ii) $h(t)=3+\cos (100 t)$; and for the same initial conditions: $x(0)=(1,-1)^{T}, v(t)=0, t \in[-\bar{h}, 0)$.

In the case i) we have $\underline{h}=0, \bar{h}=4$. Solving the LMI system (16) for $\alpha=0.2$ gives

$$
K=\left(\begin{array}{ll}
0.7889 & 3.7978
\end{array}\right) \text {. }
$$

For the case ii) the estimates of the delay are $\underline{h}=2$ and $\bar{h}=4$. The corresponding vector of feedback gains obtained by the LMI system (16) is the following

$$
K=(1.07474 .5060) \text {. }
$$

The simulations results for control of double integrator are presented on the figures 3 and 4 . They show that the control algorithm based on the interval predictor technique provides three times faster convergence rate of the system states to the origin comparing with the adaptive scheme presented in [6]. Moreover, in contrast to adaptive algorithm it shows a better dumping during the transitory motion.

The control scheme based on Luenberger-like observer application also allows us to stabilize the double inte- 


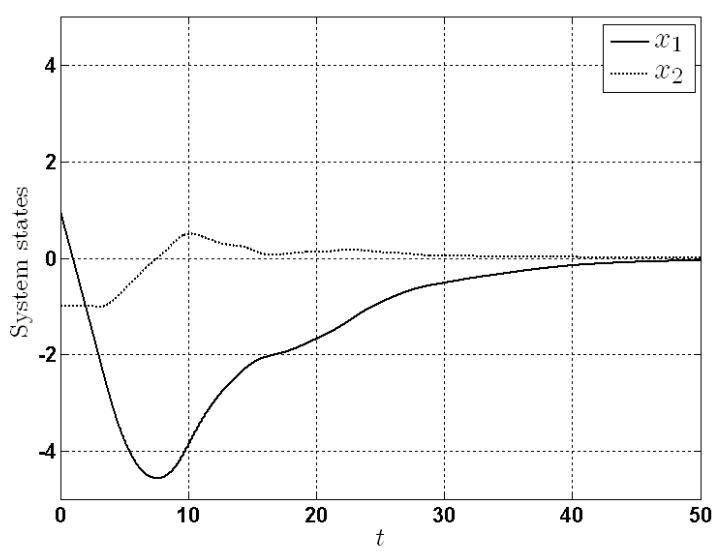

Fig. 3. Evolution of states for controlled double integrator for the case i).

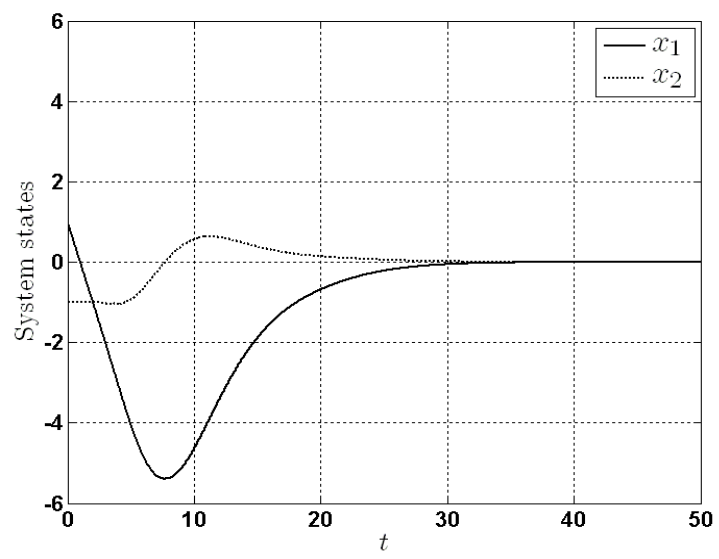

Fig. 4. Evolution of states for controlled double integrator for the case ii).

grator under the same restrictions on delay. However, it provides a slower convergence rate, which is close to the results of the paper [6].

\section{Conclusions}

The two output-based predictor feedback control algorithm are presented for exponential stabilization of a linear system with unknown time-varying input delay. The first one is based on an expansion of interval observer technique to systems with unknown time-varying input delay. The corresponding procedure of the observer design requires solving of the special Silvester's equation. The second algorithm uses the standard Luenberger-like observer. In both cases the predictor-based feedback control is designed by means of finding a solution of the LMI system. The stability analysis of closed-loop systems is based on the method of Lyapunov-Krasovskii functionals, which allow us to present the delay-depended stability conditions using upper and lower estimates of the unknown input delay. The numerical simulations show a better performance for the interval observer-based control scheme. The main disadvantage of the presented control algorithms is the necessity of on-line calculation of the integral transformation in order obtain the predictor variables (see, formulas (11), (12) and (14)). This problem is common for many predictor-based control schemes.

The main results are presented for a linear system with uncertain input delay. However, they can be possibly extended to the case of state and/or output delays and other types of system uncertainties and disturbances. It is also important to present the control design schemes, which will take into account the rate of the delay variation. The second statement of Lemma 4 guarantees that slow variation of the control input implies small observation errors. Seemingly, this property will allow us to solve a tracking problem for a system with time-varying and unknown input delay, if a reference signal will be sufficiently slow. All these problems are subjected for future researches.

\section{Appendix}

\section{Proof of Theorem 8}

I. Due to the form of the control input (15) for $t>\Delta h$, we have

$$
\begin{aligned}
& \underline{\tilde{B} u}(t)-\tilde{B} u(t)=\min _{\theta \in[0, \Delta h]}-\tilde{B} K \int_{t-\theta}^{t} \dot{z}(s) d s, \\
& \overline{\tilde{B} u}(t)-\tilde{B} u(t)=\max _{\theta \in[0, \Delta h]}-\tilde{B} K \int_{t-\theta}^{t} \dot{z}(s) d s,
\end{aligned}
$$

where $\min (\max )$ is considered in componentwise sense. Then for any function $z(t)$ there exist functions $\theta_{i}$ : $\mathbb{R}_{+} \rightarrow[0, \Delta h], i=1,2, \ldots, 2 n$ such that

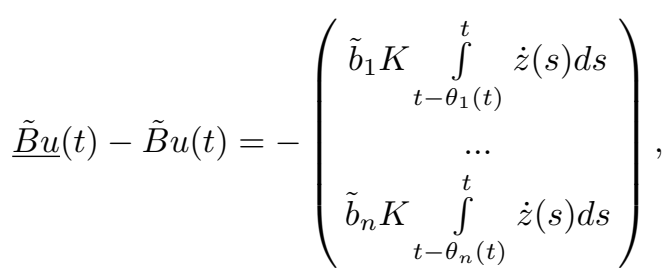

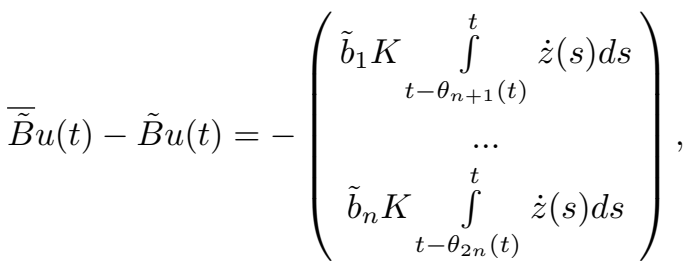

where $\tilde{b}_{i}$ is the $i$-th row of the matrix $\tilde{B}$. 
Introduce the auxiliary state vector $e=\frac{\bar{e}-\underline{e}}{2}$, where $\bar{e}=$ $\bar{x}-\tilde{x}$ and $\underline{e}=\tilde{x}-\underline{x}$. In this case using (8), (11) and (12) for $t \geq \bar{h}$ we obtain the following system

$$
\begin{aligned}
& \dot{e}=(\tilde{A}+\tilde{L} \tilde{C}) e-\sum_{i=1}^{2 n} \tilde{B}_{i} K \int_{t-\underline{h}-\theta_{i}(t-\underline{h})}^{t-h(t)} \frac{\dot{z}(s)}{2} d s, \\
& \dot{z}=(\tilde{A}+\tilde{B} K) z-\sum_{i=1}^{2 n} \tilde{B}_{i} K \int_{t-\theta_{i}(t)}^{t} \frac{\dot{z}(s)}{2} d s+e^{\tilde{A}} \underline{h} \tilde{L} \tilde{C} e .
\end{aligned}
$$

II. Consider the Lyapunov-Krasovski functional (LKF) defined for $t \geq \bar{h}$

$$
\begin{gathered}
V(e(t), z(t), \dot{z}(\cdot))=V_{e}+V_{z}+V_{e z} \\
V_{e}(e(t), \dot{z}(\cdot))=e^{T}(t) Q e(t)+ \\
\Delta h \sum_{i=1}^{2 n} \int_{-\Delta h}^{0} \int_{t-\underline{h}+\theta}^{t-\underline{h}} e^{\beta(s-t+\underline{h})} \dot{z}^{T}(s) \tilde{S}_{i} \dot{z}(s) d s d \theta \\
V_{z}(z(t), \dot{z}(\cdot))=z^{T}(t) P z(t)+ \\
\Delta h \sum_{i=1}^{2 n} \int_{-\Delta h}^{0} \int_{t+\theta}^{t} e^{\alpha(s-t)} \dot{z}^{T}(s) \tilde{R}_{i} \dot{z}(s) d s d \theta \\
V_{e z}(\dot{z}(\cdot))=(\Delta h)^{2} \sum_{i=1}^{2 n} \int_{t-\underline{h}}^{t} e^{\gamma(s-t+\underline{h})} \dot{z}^{T}(s) \tilde{S}_{i} \dot{z}(s) d s
\end{gathered}
$$

where $\alpha, \beta, \gamma \in \mathbb{R}_{+}, P, Q, \tilde{S}_{i}, \tilde{R}_{i} \in \mathbb{R}^{n \times n}, P \succ 0, Q \succ 0$, $\tilde{S}_{i} \succ 0, \tilde{R}_{i} \succ 0, i=1,2, \ldots, 2 n$.

The structure of term $V_{z}$ of the LKF $V$ is similar to the one from the paper [11]. The terms $V_{e z}$ and $V_{e}$ are motivated by the extended system (19), that implicitly contains both system state and observer state.

Remark also that the presented functional $V$ has the form of a discretized LKF [14]. Usually the discretization is applied to a complete LKF [14] in order to obtain stability conditions in LMI forms. The "discretized" LKF of this paper is imposed by structures of the interval observer and the interval predictor.

III. Calculating the time derivative of the functional $V_{z}(z(t), \dot{z}(\cdot))$ we derive

$$
\begin{gathered}
\dot{V}_{z}(z(t), \dot{z}(\cdot))=2 z^{T}(t) P \dot{z}(t)- \\
\alpha \Delta h \sum_{i=1}^{2 n} \int_{-\Delta h}^{0} \int_{t+\theta}^{t} e^{\alpha(s-t)} \dot{z}^{T}(s) \tilde{R}_{i} \dot{z}(s) d s d \theta+ \\
(\Delta h)^{2} \sum_{i=1}^{2 n} \dot{z}^{T}(t) \tilde{R}_{i} \dot{z}(t)- \\
\Delta h \sum_{i=1}^{2 n} \int_{t-\Delta h}^{t} e^{\alpha(s-t)} \dot{z}^{T}(s) \tilde{R}_{i} \dot{z}(s) d s=-\alpha V_{z}(z(t), \dot{z}(\cdot))+ \\
\alpha z^{T}(t) P z(t)+2 z^{T}(t) P \dot{z}(t)+(\Delta h)^{2} \sum_{i=1}^{2 n} \dot{z}^{T}(t) \tilde{R}_{i} \dot{z}(t)- \\
\Delta h \sum_{i=1}^{2 n} \int_{t-\Delta h}^{t} e^{\alpha(s-t)} \dot{z}^{T}(s) \tilde{R}_{i} \dot{z}(s) d s .
\end{gathered}
$$

On the one hand we have

$$
\frac{1}{e^{\alpha \Delta h}} \int_{t-\Delta h}^{t} \dot{z}^{T}(s) \tilde{R}_{i} \dot{z}(s) d s \leq \int_{t-\Delta h}^{t} e^{\alpha(s-t)} \dot{z}^{T}(s) \tilde{R}_{i} \dot{z}(s) d s .
$$

On the other hand due to $0 \leq \theta_{i}(t) \leq \Delta h$ the following inequalities

$$
\begin{gathered}
\int_{t-\theta_{i}(t)}^{t} \dot{z}^{T}(s) \tilde{R}_{i} \dot{z}(s) d s \leq \int_{t-\Delta h}^{t} \dot{z}^{T}(s) \tilde{R}_{i} \dot{z}(s) d s, \\
\theta_{i}(t) \int_{t-\theta_{i}(t)}^{t} \dot{z}^{T}(s) \tilde{R}_{i} \dot{z}(s) d s \leq \Delta h \int_{t-\theta_{i}(t)}^{t} \dot{z}^{T}(s) \tilde{R}_{i} \dot{z}(s) d s
\end{gathered}
$$

hold. So, taking into account Jensen's Inequality

$$
\begin{gathered}
\left(\int_{t-\theta_{i}(t)}^{t} \dot{z}(s) d s\right)^{T} \tilde{R}_{i}\left(\int_{t-\theta_{i}(t)}^{t} \dot{z}(s) d s\right) \leq \\
\theta_{i}(t) \int_{t-\theta_{i}(t)}^{t} \dot{z}^{T}(s) \tilde{R}_{i} \dot{z}(s) d s,
\end{gathered}
$$

we derive

$$
\begin{aligned}
& \dot{V}_{z} \leq-\alpha V_{z}+\alpha z^{T}(t) P z(t)+ \\
& (\Delta h)^{2} \dot{z}^{T}(t)\left(\sum_{i=1}^{2 n} \tilde{R}_{i}\right) \dot{z}(t)+2 z^{T}(t) P \dot{z}(t)- \\
& e^{-\alpha \Delta h} \sum_{i=1}^{2 n}\left(\int_{t-\theta_{i}(t)}^{t} \dot{z}(s) d s\right)^{T} \tilde{R}_{i}\left(\int_{t-\theta_{i}(t)}^{t} \dot{z}(s) d s\right)= \\
& -\alpha V_{z}(z(t), \dot{z}(\cdot))+g_{z}^{T} W_{z}^{1} g_{z}- \\
& (\Delta h)^{2} \dot{z}^{T}(t)\left(\sum_{i=1}^{2 n} e^{\gamma \underline{h}} \tilde{S}_{i}\right) \dot{z}(t),
\end{aligned}
$$

where

$$
\begin{gathered}
g_{z}=\left(z(t), \dot{z}(t), \int_{t-\theta_{1}(t)}^{t} \dot{z}(s) d s, \ldots \int_{t-\theta_{2 n}(t)}^{t} \dot{z}(s) d s\right)^{T}, \\
W_{z}^{1}=\left(\begin{array}{ccccc}
\alpha P & P & 0 & \ldots & 0_{n} \\
P & (\Delta h)^{2} \sum_{i=1}^{2 n} \tilde{R}_{i}+e^{\gamma \underline{h}} \tilde{S}_{i} & 0_{n} & \ldots & 0_{n} \\
0_{n} & 0_{n} & \frac{-1}{e^{\alpha \Delta h}} \tilde{R}_{1} & \ldots & 0_{n} \\
\ldots & \ldots & \ldots & \ldots & \ldots \\
0_{n} & 0_{n} & 0_{n} & \ldots & \frac{-1}{e^{\alpha \Delta h}} \tilde{R}_{2 n}
\end{array}\right) .
\end{gathered}
$$

Similar considerations for the functional $V_{e}(e(t), \dot{z}(\cdot))$ 
give the following estimate

$$
\begin{gathered}
\dot{V}_{e} \leq-\beta V_{e}+\beta e^{T}(t) Q e(t)+2 e^{T}(t) Q \dot{e}(t)+ \\
(\Delta h)^{2} \dot{z}^{T}(t-\underline{h})\left(\sum_{i=1}^{2 n} \tilde{S}_{i}\right) \dot{z}(t-\underline{h})- \\
\frac{1}{e^{\beta \Delta h}} \sum_{i=1}^{2 n}\left(\int_{t-\underline{h}-\theta_{i}(t-\underline{h})}^{t-h(t)} \dot{z}(s) d s\right)^{T} \tilde{S}_{i}\left(\int_{t-\underline{h}-\theta_{i}(t-\underline{h})}^{t-h(t)} \dot{z}(s) d s\right)= \\
-\beta V_{e}(z(t), \dot{z}(\cdot))+g_{e}^{T} W_{e}^{1} g_{e}+2 e^{T}(t) Q \dot{e}(t)+ \\
(\Delta h)^{2} \dot{z}^{T}(t-\underline{h})\left(\sum_{i=1}^{2 n} \tilde{S}_{i}\right) \dot{z}(t-\underline{h}),
\end{gathered}
$$

where

$$
\begin{gathered}
g_{e}=\left(e(t), \int_{t-\underline{h}-\theta_{1}(t-\underline{h})}^{t-\underline{h}} \dot{z}(s) d s, \ldots, \int_{t-\underline{h}-\theta_{2 n}(t-\underline{h})}^{t-\underline{h}} \dot{z}(s) d s\right)^{T}, \\
W_{e}^{1}=\left(\begin{array}{cccc}
\beta Q & 0_{n} & \ldots & 0_{n} \\
0_{n} & -e^{-\beta \Delta h} \tilde{S}_{1} & \ldots & 0_{n} \\
\ldots & \ldots & \ldots & \ldots \\
0_{n} & \ldots & \ldots-e^{-\beta \Delta h} \tilde{S}_{2 n}
\end{array}\right) .
\end{gathered}
$$

Finally, taking into account that

$$
\begin{gathered}
\frac{d}{d t} \int_{t-\underline{h}}^{t} e^{\gamma(s-t+\underline{h})} \dot{z}^{T}(s) \tilde{S}_{i} \dot{z}(s) d s=e^{\gamma \underline{h}} \dot{z}^{T}(t) \tilde{S}_{i} \dot{z}(t)- \\
\gamma \int_{t-\underline{h}}^{t} e^{\gamma(s-t+\underline{h})} \dot{z}^{T}(s) \tilde{S}_{i} \dot{z}(s) d s-\dot{z}^{T}(t-\underline{h}) \tilde{S}_{i} \dot{z}(t-\underline{h})
\end{gathered}
$$

we conclude

$$
\begin{gathered}
\dot{V}(e(t), z(t), \dot{z}(\cdot)) \leq-\min \{\alpha, \beta, \gamma\} V(e(t), z(t), \dot{z}(\cdot))+ \\
\left(\begin{array}{l}
g_{e} \\
g_{z}
\end{array}\right)^{T}\left(\begin{array}{cc}
W_{e}^{1} & 0 \\
0 & W_{z}^{1}
\end{array}\right)\left(\begin{array}{l}
g_{e} \\
g_{z}
\end{array}\right)+2 e^{T}(t) Q \dot{e}(t) .
\end{gathered}
$$

IV. Following the descriptor approach [10] we consider the following equality

$$
\begin{array}{r}
0=2 e^{T} Q\left((\tilde{A}+\tilde{L} \tilde{C}) e-\sum_{i=1}^{2 n} \tilde{B}_{i} K \int_{t-\underline{h}-\theta_{i}(t-\underline{h})}^{t-h(t)} \frac{\dot{z}(s)}{2} d s-\dot{e}\right) \\
+2(P z+\Delta h P \dot{z})^{T} \times \\
\left(\begin{array}{c}
\left.(\tilde{A}+\tilde{B} K) z-\sum_{i=1}^{2 n} \tilde{B}_{i} K \int_{t-\theta_{i}(t)}^{t} \frac{\dot{z}(s)}{2} d s+e^{\tilde{A}} \underline{h} \tilde{L} \tilde{C} e-\dot{z}\right)
\end{array}\right.
\end{array}
$$

that obviously holds for any solution $(e(t), z(t))$ of the system (19) if $t>\bar{h}$. This equality can be rewritten in the form

$$
0=\left(\begin{array}{l}
g_{e} \\
g_{z}
\end{array}\right)^{T}\left(\begin{array}{cc}
W_{e}^{2} & W_{0} \\
W_{0}^{T} & W_{z}^{2}
\end{array}\right)\left(\begin{array}{c}
g_{e} \\
g_{z}
\end{array}\right)-2 e^{T}(t) Q \dot{e}(t)
$$

where

$$
\begin{gathered}
W_{e}^{2}=\left(\begin{array}{cccc}
Q(\tilde{A}+\tilde{L} \tilde{C})+(\tilde{A}+\tilde{L} \tilde{C})^{T} Q & \frac{-Q \tilde{B}_{1} K}{2} & \ldots & \frac{-Q \tilde{B}_{2 n} K}{2} \\
\frac{-K^{T} \tilde{B}_{1}^{T} Q}{2} & 0_{n} & \ldots & 0_{n} \\
\ldots & \ldots & \ldots & \ldots \\
\frac{-K^{T} \tilde{B}_{2 n}^{T} Q}{2} & 0_{n} & \ldots & 0_{n}
\end{array}\right), \\
W_{z}^{2}=\left(\begin{array}{ccccc}
\Psi_{1} & \Psi_{2} & \frac{-P \tilde{B}_{1} K}{2} & \ldots & \frac{-P \tilde{B}_{2 n} K}{2} \\
\frac{\Psi_{2}^{T}}{-K^{T} \tilde{B}_{1}^{T} P} & -2 \Delta h P & \frac{-P \tilde{B}_{1} K}{2(\Delta h)^{-1}} & \ldots & \frac{-P \tilde{B}_{2 n} K}{2(\Delta h)^{-1}} \\
\ldots & \frac{-K^{T} \tilde{B}_{1}^{T} P}{2(\Delta h)^{-1}} & 0_{n} & \ldots & 0_{n} \\
\ldots & \ldots & \ldots & \ldots \\
\frac{-K^{T} \tilde{B}_{2 n}^{T} P}{2} & \frac{-K^{T} \tilde{B}_{2 n}^{T} P}{2(\Delta h)^{-1}} & 0_{n} & \ldots & 0_{n}
\end{array}\right), \\
W_{0}=\left(\begin{array}{cccc}
\tilde{C}^{T} \tilde{L}^{T} e^{\tilde{A}^{T} \underline{h}[P} & \Delta h P] & 0_{n \times\left(2 n^{2}\right)} \\
0_{\left(2 n^{2}\right) \times 2 n} & & 0_{2 n^{2}}
\end{array}\right)
\end{gathered}
$$

where $\Psi_{1}=P(\tilde{A}+\tilde{B} K)+(\tilde{A}+\tilde{B} K)^{T} P$ and $\Psi_{2}=$ $\Delta h(\tilde{A}+\tilde{B} K)^{T} P-P$. Hence, the time derivative of the functional $V$ calculated along the trajectories of the system (19) can be estimated as

$$
\begin{gathered}
\dot{V}(e(t), z(t), \dot{z}(\cdot)) \leq-\min \{\alpha, \beta, \gamma\} V(e(t), z(t), \dot{z}(\cdot))+ \\
\left(\begin{array}{c}
g_{e} \\
g_{z}
\end{array}\right)^{T}\left(\begin{array}{cc}
W_{e}^{1}+W_{e}^{2} & W_{0} \\
W_{0}^{T} & W_{z}^{1}+W_{z}^{2}
\end{array}\right)\left(\begin{array}{c}
g_{e} \\
g_{z}
\end{array}\right) .
\end{gathered}
$$

Finally, for $\beta=\gamma=\alpha$ and

$$
Z=Q^{-1}, X=P^{-1}, \tilde{S}_{i}=\frac{1}{4} P S_{i} P, \tilde{R}_{i}=\frac{1}{4} P R_{i} P, Y=K P^{-1},
$$

we have

$$
G^{T}\left(\begin{array}{cc}
W_{e}^{1}+W_{e}^{2} & W_{0} \\
W_{0}^{T} & W_{z}^{1}+W_{z}^{2}
\end{array}\right) G=\left(\begin{array}{cc}
W_{e} & W_{e z} \\
W_{e z}^{T} & W_{z}
\end{array}\right)
$$

where

$G=\left(\begin{array}{ccccc}I_{n} & 0 & 0 & 0 & 0 \\ 0 & -2 I_{n^{2}} & 0 & 0 & 0 \\ 0 & 0 & I_{n} & 0 & 0 \\ 0 & 0 & 0 & \frac{1}{\Delta h} I_{2 n} & 0 \\ 0 & 0 & 0 & 0 & -2 I_{4 n^{2}}\end{array}\right)\left(\begin{array}{cccc}Q^{-1} & 0 & \ldots & 0 \\ 0 & P^{-1} & \ldots & 0 \\ \ldots & \ldots & \ldots & \ldots \\ 0 & 0 & \ldots & P^{-1}\end{array}\right)$ 
Therefore, feasibility of LMI (16) implies exponential stability of (19).

V. By the definition we have

$$
\begin{gathered}
e(t)=\frac{\bar{e}(t)-\underline{e}(t)}{2}=\frac{\bar{x}(t)+\underline{x}(t)}{2}-\tilde{x}(t) \\
z(t)=\frac{\bar{z}(t)+\underline{z}(t)}{2}=e^{\tilde{A} \underline{h}} \frac{\underline{x}(t)+\bar{x}(t)}{2}+ \\
\int_{-\underline{h}}^{0} e^{-\tilde{A} \theta}\left(\tilde{B} u(t+\theta)+\frac{\Delta \tilde{B} u(t+\theta)+\bar{\Delta} \tilde{B} u(t+\theta)}{2}\right) d \theta
\end{gathered}
$$

where $u(t)=K z(t)$. Hence, the limits $e(t) \rightarrow 0$ and $z(t) \rightarrow 0$ imply $\tilde{x}(t) \rightarrow 0$ or, equivalently, $x(t) \rightarrow 0$. It can be easily shown that the rate of convergence of $x(t)$ to zero is the same as for $e(t)$ and $z(t)$. The functional $V$ calculated along trajectories of the system (19) tends to zero exponentially with the rate $\alpha$. Since we have $\|e\| \leq \lambda_{\min }^{-1}(Q) \sqrt{V}$ and $\|z\| \leq \lambda_{\min }^{-1}(P) \sqrt{V}$ then $z(t)$ and $e(t)$ converge to zero exponentially with a rate $p$, which is greater than or equal to $\alpha / 2$.

Since $u(t)=K z(t)$ converges to zero exponentially with the rate $p \geq \alpha / 2$ and $\alpha / 2 \in\left(0,-\max _{\lambda \in \operatorname{spec}(\tilde{A}+\tilde{L} \tilde{C})} \Re(\lambda)\right)$ (see, the considerations after the statement of Theorem 8), then selecting $q \in\left(\frac{\alpha}{2},-\max _{\lambda \in \operatorname{spec}(\tilde{A}+\tilde{L} \tilde{C})} \Re(\lambda)\right)$ by the second statement of Lemma 4 we derive that $\bar{e}(t)$ and $\underline{e}(t)$ converges to zero exponentially with the rate $\min \{p, q\} \geq \alpha / 2$.

\section{Proof of Theorem 10}

I. Denote $\hat{e}=\hat{x}-x$. Then the system (1) with the observer (13) and the control $u(t)=\mathcal{K} z_{l}(t)$ can be rewritten in the form

$$
\left\{\begin{array}{l}
\dot{\hat{e}}=(A+\mathcal{L} C) \hat{e}+B \mathcal{K} \int_{t-h(t)}^{t-\underline{h}} \dot{\hat{z}}(s) d s \\
\dot{\hat{z}}=(A+B \mathcal{K}) \hat{z}+e^{A} \underline{h} \mathcal{L} C \hat{e}
\end{array}\right.
$$

where $\hat{z}$ is defined by the formula (14). Obviously that $\hat{z}(t) \rightarrow 0$ and $\hat{e}(t) \rightarrow 0$ imply $x(t) \rightarrow 0$.

II. The rest part of the proof is just sketched, since it repeats the considerations presented in the proof of Theorem 8. Consider the Lyapunov-Krasovski functional of the form

$$
\begin{gathered}
V(\hat{e}(t), \hat{z}(t), \dot{\hat{z}}(\cdot))= \\
e^{T}(t) \mathcal{Q} e(t)+z^{T}(t) \mathcal{P} z(t)+V_{1}(\dot{\hat{z}}(\cdot))+V_{2}(\dot{\hat{z}}(\cdot)), \\
V_{1}(\dot{\hat{z}}(\cdot))=(\Delta h)^{2} \int_{t-\underline{h}}^{t} e^{\gamma(s-t+\underline{h})} \dot{\hat{z}}^{T}(s) \tilde{\mathcal{R}} \dot{\hat{z}}(s) d s, \\
V_{2}(\dot{\hat{z}}(\cdot))=\Delta h \int_{-\Delta h t-\underline{h}+\theta}^{0} \int^{t-\underline{h}} e^{\mu(s-t+\underline{h})} \dot{\hat{z}}^{T}(s) \tilde{\mathcal{R}} \dot{\hat{z}}(s) d s d \theta,
\end{gathered}
$$

where $\gamma, \mu \in \mathbb{R}_{+}, \mathcal{Q}, \mathcal{P}, \tilde{\mathcal{R}} \in \mathbb{R}^{2 n \times 2 n}, \mathcal{P} \succ 0, \mathcal{Q} \succ 0$, $\tilde{\mathcal{R}} \succ 0$.

The time derivative of the functional $V$ can be estimated as follows

$$
\begin{aligned}
& \dot{V}(\hat{e}(t), \hat{z}(t), \dot{\hat{z}}(\cdot)) \leq-\alpha \hat{e}^{T}(t) \mathcal{Q} \hat{e}(t)-\beta \hat{z}^{T}(t) \mathcal{P} \hat{z}(t) \\
& -\gamma V_{1}(\dot{\hat{z}}(\cdot))-\mu V_{2}(\dot{\hat{z}}(\cdot))+2 \hat{e}^{T}(t) \mathcal{Q} \dot{\hat{e}}(t)+g^{T} W_{1} q
\end{aligned}
$$

where $\alpha, \beta \in \mathbb{R}_{+}$and

$$
\begin{gathered}
g=\left(\hat{e}(t), \hat{z}(t), \dot{\hat{z}}(t), \int_{t-h(t)}^{t-\underline{h}} \dot{\hat{z}}(s) d s\right)^{T}, \\
W_{1}=\left(\begin{array}{cccc}
\alpha \mathcal{Q} & 0 & 0 & 0 \\
0 & \beta \mathcal{P} & \mathcal{P} & 0 \\
0 & \mathcal{P} & (\Delta h)^{2} e^{\gamma \underline{h} \tilde{\mathcal{R}}} & 0 \\
0 & 0 & 0 & -e^{-\mu \Delta h} \tilde{\mathcal{R}}
\end{array}\right) .
\end{gathered}
$$

III. We consider the following equality

$0=2 \hat{e}^{T}(t) \mathcal{Q}\left((A+\mathcal{L} C) \hat{e}(t)+B \mathcal{K} \int_{t-h(t)}^{t-\underline{h}} \dot{\hat{z}}(s) d s-\dot{\hat{e}}(t)\right)+$

$2(\mathcal{P} \hat{z}(t)+\Delta h \mathcal{P} \dot{\hat{z}}(t))^{T}\left((A+B \mathcal{K}) \hat{z}(t)+e^{A} \underline{h} \mathcal{L} C \hat{e}(t)-\dot{\hat{z}}(t)\right)$, that obviously holds for any solution $(e(t), z(t))$ of the system (20) if $t>\bar{h}$. This equality can be rewritten in the form $0=g^{T} W_{2} g$, where

$$
W_{2}=\left(\begin{array}{cccc}
\tilde{\Pi}_{11} & \tilde{\Pi}_{12} & \tilde{\Pi}_{13} & \tilde{\Pi}_{14} \\
\tilde{\Pi}_{12}^{T} & \tilde{\Pi}_{22} & \tilde{\Pi}_{23} & 0 \\
\tilde{\Pi}_{13}^{T} & \tilde{\Pi}_{23} & -\Delta h \mathcal{P} & 0 \\
\tilde{\Pi}_{14}^{T} & 0 & 0 & 0
\end{array}\right)
$$

with $\tilde{\Pi}_{11}=\mathcal{Q}(A+\mathcal{L} C)+(A+\mathcal{L} C)^{T} \mathcal{Q}, \tilde{\Pi}_{12}=$ $C^{T} \mathcal{L}^{T} e^{A^{T}} \underline{h} \mathcal{P}, \tilde{\Pi}_{22}=\mathcal{P}(A+B \mathcal{K})+(A+B \mathcal{K})^{T} \mathcal{P}$, 
$\tilde{\Pi}_{13}=\Delta h C^{T} \mathcal{L}^{T} e^{A^{T} \underline{h}} \mathcal{P}, \tilde{\Pi}_{23}=\Delta h \mathcal{P}(A+B \mathcal{K})-\mathcal{P}$, $\tilde{\Pi}_{14}=\mathcal{Q B K}$.

Hence, for $t>\underline{h}$ the time derivative of the functional $V$ calculated along the trajectories of the system (20) can be over-estimated as

$$
\dot{V}(\hat{e}(t), \hat{z}(t), \dot{\hat{z}}(\cdot)) \leq-2 r V(\hat{e}(t), \hat{z}(t), \dot{\hat{z}}(\cdot))+g^{T}\left(W_{1}+W_{2}\right) q,
$$

where $r=0.5 \min \{\alpha, \beta, \gamma, \mu\}$.

Finally, for $\mu=\gamma=\beta=\alpha$ and

$$
\mathcal{Z}=\mathcal{Q}^{-1}, \mathcal{X}=\mathcal{P}^{-1}, \tilde{\mathcal{R}}=e^{-\gamma \underline{h}} \mathcal{P} \mathcal{R} \mathcal{P}, \mathcal{Y}=\mathcal{K} \mathcal{P}^{-1}
$$

we have

$$
G^{T}\left(W_{1}+W_{2}\right) G=\mathcal{W},
$$

where $G=\operatorname{diag}\left\{\mathcal{Q}^{-1}, \mathcal{P}^{-1}, \mathcal{P}^{-1}, \mathcal{P}^{-1}\right\}$.

\section{References}

[1] Z. Artstein. Linear systems with delayed controls: A reduction. IEEE Transactions on Automatic Control, 27(4):869-879, 1982.

[2] R. H. Bartels and G. W. Stewart. Solution of the matrix equation $\mathrm{AX}+\mathrm{XB}=\mathrm{C}$. Communications of the ACM, 15:820-826, 1972.

[3] N. Bekiaris-Liberis and M. Krstic. Compensation of timevarying input and state delays for nonlinear systems. Journal of Dynamic Systems, Measurement, and Control, 134(1):paper 011009, 2012.

[4] L. Belkoura, J.-P. Richard, and M. Fliess. Parameters estimation of systems with delayed and structured entries. Automatica, 45(5):1117-1125, 2009.

[5] D. Bresch-Pietri and M. Krstic. Adaptive trajectory tracking despite unknown input delay and plant parameters. Automatica, 45(9):2074-2081, 2009.

[6] H.-L. Choi and J.-T. Lim. Output feedback regulation of a chain of integrators with an unknown time-varying delay in the input. IEEE Transactions on Automatic Control, 55(1):263-268, 2010.

[7] D. Efimov, W. Perruquetti, and J.-P. Richard. Interval estimation for uncertain systems with time-varying delays. International Journal of Control, (accepted), 2013.

[8] D. Efimov, T. Raissi, and A. Zolghadri. Control of nonlinear and LPV systems: interval observer-based framework. IEEE Transactions on Automatic Control, 57:to appear, 2012.

[9] L. Farina and S. Rinaldi. Positive Linear Systems: Theory and Applications. New York: Wiley Interscience, 2000.

[10] E. Fridman. Descriptor discretized lyapunov functional method: Analysis and design. IEEE Transactions on Automatic Control, 51(5):890-897, 2006.

[11] E. Fridman. A refined input delay approach to sampled-data control. Autom, 46(2):421-427, 2010.

[12] E. Fridman, A. Seuret, and J.-P. Richard. Robust sampleddata stabilization of linear systems: an input delay approach. Automatica, 40(8):1441-1446, 2004.

[13] J.L. Gouze, A. Rapaport, and M.Z. Hadj-Sadok. Interval observers for uncertain biological systems. Ecological Modeling, 133(1):46-56, 2000.
[14] K. Gu, V. Kharitonov, and J. Chen. Stability of Time-Delay Systems. Boston, MA: Birkhauser, 2003.

[15] M. Jankovic. Recursive predictor design for state and output feedback controllers for linear time delay systems. Automatica, 46(3):510-517, 2010.

[16] M. Krstic. Delay Compensation for Nonlinear, Adaptive, and PDE Systems. Birkhauser Boston, 2009.

[17] A. Kruszewski, W. J. Jiang, E. Fridman, J.-P. Richard, and A. Toguyeni. A switched system approach to exponential stabilization through communication network. IEEE Transactions on Control Systems Technology, 20(4):887-900, 2012.

[18] F. Mazenc and O. Bernard. Interval observers for linear timeinvariant systems with disturbances. Automatica, 47(1):140$147,2011$.

[19] F. Mazenc, S. Niculescu, and M. Krstic. Lyapunov-Krasovskii functionals and application to input delay compensation for linear time-invariant systems. Automatica, 48(7):1317-1323, 2012.

[20] F. Mazenc, S.-I. Niculescu, and O. Bernard. Exponentially stable interval observers for linear systems with delay. SIAM Journal of Control and Optimization, 50:285-315, 2012.

[21] A. Polyakov. Minimization of disturbances effects in time delay predictor-based sliding mode control systems. Journal of The Franklin Institute, 349(4):1380-1396, 2012.

[22] T. Raissi, D. Efimov, and A. Zolghadri. Interval state estimation for a class of nonlinear systems. IEEE Transactions on Automatic Control, 57(1):260-265, 2012.

[23] J.-P. Richard. Time-delay systems: An overview of some recent advances and open problems. Automatica, 39(10):1667-1694, 2003.

[24] A. Seuret, T. Floquet, J.-P. Richard, and S. K. Spurgeon. A sliding mode observer for linear systems with unknown time varying delay. In American Control Conference, pages 4558-4563, 2007.

[25] O. J. M. Smith. A controller to overcome dead time. Journal of Instrument Society of America, 6:28-33, 1959.

[26] A. Teel, D. Nesic, and P. V. Kokotovic. A note on inputto-state stability of sampled-data nonlinear systems. In Proceedings of the IEEE Conference on Decision and Control, pages 2473-2478, 1998.

[27] K. Watanabe. Finite spectrum assignment and observer for multivariable systems with commensurate delays. IEEE Transactions on Automatic Control, 31(6):543-550, 1986.

[28] E. Witrant, C. Canudas-de Wit, D. Georges, and M. Alamir. Remote stabilization via communication networks with a distributed control law. IEEE Transactions on Automatic Control, 52(8):1480-1485, 2007.

[29] D. Yue and Q.-L. Han. Delayed feedback control of uncertain systems with time-varying input delay. Automatica, 41(2):233-240, 2005. 